\title{
NUMERICAL INVARIANTS OF IDENTITIES OF UNITAL ALGEBRAS
}

\author{
DUŠAN REPOVŠ AND MIKHAIL ZAICEV
}

\begin{abstract}
We study polynomial identities of algebras with adjoined external unit. For a wide class of algebras we prove that adjoining external unit element leads to increasing of PI-exponent precisely to 1 . We also show that any real number from the interval $[2,3]$ can be realized as PI-exponent of some unital algebra.
\end{abstract}

\section{INTRODUCTION}

We study numerical characteristics of polynomial identities of algebras over a field $F$ of characteristic zero. Given an algebra $A$ over $F$, one can associate to it the sequence $\left\{c_{n}(A)\right\}$ of non-negative integers called the sequence of codimensions. If the growth of $\left\{c_{n}(A)\right\}$ is exponential then the limiting ration of consecutive terms is called PI-exponent of $A$ and written $\exp (A)$. In the present paper we are mostly interested what happens with PI-exponent if we adjoin to $A$ an external unit element.

The first results in this area were proved for associative algebras. It is known that $\exp (A)$ is an integer in the associative case [6], [7]. It was shown in [9] that it follows from the proofs in [6], [7] that either $\exp \left(A^{\sharp}\right)=\exp (A)$ or $\exp \left(A^{\sharp}\right)=\exp (A)+1$ and both options can be realized. Here $A^{\sharp}$ is the algebra $A$ with adjoined external unit.

The next result was published in [15], following an example of A 5-dimensional algebra $A$ with $\exp (A)<2$ constructed in [4]. The point is that in the associative or Lie case PI-exponent cannot be less than 2 ([11], [13]). For a finite dimensional Lie superalgebra, Jordan and alternative algebra PI-exponent is also at least 2. Starting from the example $A$ from [4] it was shown in [15] that $\exp \left(A^{\sharp}\right)=\exp (A)+1$. In [15] also the following problem was stated: is it true that always either $\exp \left(A^{\sharp}\right)=$ $\exp (A)$ or $\exp \left(A^{\sharp}\right)=\exp (A)+1$ ?

An example of 4-dimensional simple algebra $A$ with a fractional PI-exponent was constructed in [2]. It was also shown that $\exp \left(A^{\sharp}\right)=\exp (A)+1$. This result was announced in [1]. It was also shown in [1] that if $A$ is itself a unital algebra then $\exp \left(A^{\sharp}\right)=\exp (A)$.

In the present paper (see Theorem 1 ) we shall prove that for a previously known series of algebras $A_{\alpha}$ with $\exp \left(A_{\alpha}\right)=\alpha, \alpha \in \mathbb{R}, 1<\alpha<2$ (see [3]) any extended algebra $A_{\alpha}^{\sharp}$ has exponent $\alpha+1$. That is, we shall show that there exist infinitely many algebras $A$ such that $\exp \left(A^{\sharp}\right)=\exp (A)+1$.

2010 Mathematics Subject Classification. Primary 16R10; Secondary 16P90.

Key words and phrases. Polynomial identity, non-associative unital algebra, codimension, exponential growth, fractional PI-exponent. 
Another important question is the following: which real numbers can be realized as PI-exponents of some algebra? For example, if $A$ is any associative PI-algebra or a finite dimensional Lie or Jordan algebra then $\exp (A)$ is an integer (see [6], [7], [14], [5]).

For unital algebras it is only known that if $\operatorname{dim} A<\infty$ then $\exp (A)$ cannot be less than 2. As a consequence of the main result of our paper (see Corollary 1 ) we shall obtain that for any real $\alpha \in[2,3]$ there exists a unital algebra $B_{\alpha}$ such that $\exp \left(B_{\alpha}\right)=\alpha$.

\section{Preliminaries}

Let $A$ be an algebra over a field $F$ of characteristic zero and let $F\{X\}$ be absolutely free algebra over $F$ with a countable set of generators $X=\left\{x_{1}, x_{2}, \ldots\right\}$. Recall that a polynomial $f=f\left(x_{1}, \ldots, x_{n}\right)$ is said to be an identity of $A$ if $f\left(a_{1} \ldots, a_{n}\right)=0$ for all $a_{1}, \ldots, a_{n} \in A$. The set $\operatorname{Id}(A)$ of all polynomial identities of $A$ forms an ideal of $F\{X\}$.

Denote by $P_{n}$ the subspace of all multilinear polynomials in $F\{X\}$ on $x_{1}, \ldots, x_{n}$. Then the intersection $\operatorname{Id}(A) \cap P_{n}$ is the space of all multilinear identities of $A$ of degree $n$.

Denote

A non-negative integer

$$
P_{n}(A)=\frac{P_{n}}{I d(A) \cap P_{n}} .
$$

$$
c_{n}(A)=\operatorname{dim} P_{n}(A)
$$

is called the $n$th codimension of $A$. Asymptotic behavior of the sequence $\left\{c_{n}(A)\right\}, n=$ $1,2, \ldots$, is an important numerical invariant of identities of $A$. We refer readers to [8] for an account of basic notions of the theory of codimensions of PI-algebras.

If the sequence $\left\{c_{n}(A)\right\}$ is exponentially bounded, i.e. $c_{n}(A) \leq a^{n}$ for all $n$ and for some number $a$ (for example in case $\operatorname{dim} A<\infty$ and in many other cases), we can define the lower and the upper PI-exponents of $A$ by

$$
\underline{\exp }(A)=\liminf _{n \rightarrow \infty} \sqrt[n]{c_{n}(A)}, \quad \overline{\exp }(A)=\limsup _{n \rightarrow \infty} \sqrt[n]{c_{n}(A)}
$$

and (the ordinary) PI-exponent

$$
\exp (A)=\lim _{n \rightarrow \infty} \sqrt[n]{c_{n}(A)}
$$

provided that $\exp (A)=\overline{\exp }(A)$.

In order to compute the values of codimensions we can consider symmetric group action on $P_{n}$ defined by

$$
\sigma f\left(x_{1}, \ldots, x_{n}\right)=f\left(x_{\sigma(1)}, \ldots, x_{\sigma(n)} \quad \forall \sigma \in S_{n} .\right.
$$

The subspace $P_{n} \cap \operatorname{Id}(A)$ is invariant under this action and we can study the structure of $P_{n}(A)$ as an $S_{n}$-module. Denote by $\chi_{n}(A)$ the $S_{n}$-character of $P_{n}(A)$ called the $n$th cocharacter of $A$. Since char $F=0$ and any $S_{n}$-representation is completely reducible, the $n$th cocharacter has the decomposition

$$
\chi_{n}(A)=\sum_{\lambda \vdash n} m_{\lambda} \chi_{\lambda}
$$

where $\chi_{\lambda}$ is the irreducible $S_{n}$-character corresponding to the partition $\lambda \vdash n$ and non-negative integer $m_{\lambda}$ is the multiplicity of $\chi_{\lambda}$ in $\chi_{n}(A)$. 
Obviously, it follows from (1) that

$$
c_{n}(A)=\sum_{\lambda \vdash n} m_{\lambda} \operatorname{deg} \chi_{\lambda} .
$$

Another important numerical characteristic is the $n$th colength of $A$ defined by

$$
l_{n}(A)=\sum_{\lambda \vdash n} m_{\lambda}
$$

with $m_{\lambda}$ taken from (1). In particular, if the sequence $\left\{l_{n}(A)\right\}$ is polynomially bounded as a function of $n$ while some of $\operatorname{deg} \chi_{\lambda}$ with $m_{\lambda} \neq 0$ are exponentially large, the principal part of the asymptotic of $\left\{c_{n}(A)\right\}$ is defined by the largest value of $\operatorname{deg} \chi_{\lambda}$ with non-zero multiplicity.

For studying asymptotic of codimensions it is convenient to use the following functions. Let $0 \leq x_{1}, \ldots, x_{d} \leq 1$ be real numbers such that $x_{1}+\cdots+x_{d}=1$. Denote

$$
\Phi\left(x_{1}, \ldots, x_{d}\right)=\frac{1}{x_{1}^{x_{1}} \cdots x_{d}^{x_{d}}} .
$$

If $d=2$ then instead of $\Phi\left(x_{1}, x_{2}\right)$ we will write

$$
\Phi_{0}(x)=\frac{1}{x^{x}(1-x)^{1-x}} .
$$

We assume that some of $x_{1}, \ldots, x_{d}$ can have zero values. In this case we assume that $0^{0}=1$.

Given $\lambda=\left(l_{1}, \ldots, \lambda_{d}\right) \vdash n$ we define

$$
\Phi(\lambda)=\frac{1}{\left(\frac{\lambda_{1}}{n}\right)^{\frac{\lambda_{1}}{n}} \cdots\left(\frac{\lambda_{d}}{n}\right)^{\frac{\lambda_{d}}{n}}} .
$$

For partitions $\lambda=\left(\lambda_{1}, \ldots, \lambda_{k}\right) \vdash n$ with $k<d$ we also consider $\Phi(\lambda)$ as in (2), assuming $\lambda_{k+1}=\cdots=\lambda_{d}=0$.

The relationship between $\operatorname{deg} \chi_{\lambda}$ and $\Phi(\lambda)$ is given by the following lemma.

Lemma 1. (see [10, Lemma 1]) Let $\lambda=\left(\lambda_{1}, \ldots, \lambda_{k}\right) \vdash n$ be a partition of $n$. If $k \leq d$ and $n \geq 100$ then

$$
\frac{\Phi(\lambda)^{n}}{n^{d^{2}+d}} \leq \operatorname{deg} \chi_{\lambda} \leq n \Phi(\lambda)^{n}
$$

Now we investigate how the value of $\Phi\left(x_{1}, \ldots, x_{d}\right)$ increases after adding one extra variable.

Lemma 2. Let

$$
\Phi\left(x_{1}, \ldots, x_{d}\right)=\frac{1}{x_{1}^{x_{1}} \cdots x_{d}^{x_{d}}}, \quad 0 \leq x_{1}, \ldots, x_{d}, \quad x_{1}+\cdots+x_{d}=1,
$$

and let $\Phi\left(z_{1}, \ldots, z_{d}\right)=$ a for some fixed $z_{1}, \ldots, z_{d}$. Then

$$
\max _{0 \leq t \leq 1}\left\{\Phi\left(y_{1}, \ldots y_{d}, 1-t\right) \mid y_{1}=t z_{1}, \ldots, y_{d}=t z_{d}\right\}=a+1 .
$$

Moreover, the maximal value is achieved if $t=\frac{a}{a+1}$. 
Proof. Consider

$$
g(t)=\ln \Phi^{-1}\left(t z_{1}, \ldots, t z_{d}, 1-t\right)
$$

Then

$$
g(t)=t \ln t+(1-t) \ln (1-t)-t \ln a .
$$

Hence its derivative is equal to

$$
g^{\prime}(t)=\ln \frac{t}{(1-t) a}
$$

and $g^{\prime}(t)=0$ if and only if $t=(1-t) a$, that is $t=\frac{a}{a+1}$. It is not difficult to check that $g$ has the minimum at this point.

Now we compute the value of $g$ :

$$
g\left(\frac{a}{a+1}\right)=\frac{a}{a+1} \ln \frac{a}{a+1}+\frac{1}{a+1} \ln \frac{1}{a+1}-\frac{a}{a+1} \ln a=\ln B,
$$

where

$$
B=\left(\frac{a}{a+1}\right)^{\frac{a}{a+1}}\left(\frac{1}{a+1}\right)^{\frac{1}{a+1}} a^{-\frac{a}{a+1}}=\frac{1}{a+1} .
$$

Hence $\Phi_{\max }=B^{-1}=a+1$, and we have completed the proof.

Next lemma shows what happens with $\Phi(\lambda)$ when we insert an extra row in Young diagram $D_{\lambda}$.

Lemma 3. Let $\gamma$ be a positive real number and let $\lambda=\left(\lambda_{1}, \ldots, \lambda_{d}\right)$ be a partition of $n$ such that $\frac{\lambda_{1}}{n}, \ldots, \frac{\lambda_{d}}{n} \geq \gamma$. Then for any $\varepsilon>0$ there exist $n^{\prime}=k n$ and a partition $\mu \vdash n^{\prime}, \mu=\left(\mu_{1}, \ldots, \mu_{d+1}\right)$ such that for some integers $1 \leq i \leq d+1$ and $q \geq 1$ the following conditions hold:

1) $\mu_{j}=q \lambda_{j}$ for all $j \leq i-1$;

2) $\mu_{j+1}=q \lambda_{j}$ for all $j \geq i$; and

3) $|\Phi(\lambda)-\Phi(\mu)+1|<\varepsilon$.

Moreover, $k$ does not depend on $\lambda$ and $n$.

Proof. Denote

$$
z_{1}=\frac{\lambda_{1}}{n}, \ldots, z_{d}=\frac{\lambda_{d}}{n}
$$

and $a=\Phi\left(z_{1}, \ldots, z_{d}\right)=\Phi(\lambda)$. By Lemma 2

$$
\Phi\left(t z_{1}, \ldots, t z_{d}, 1-t\right)=a+1
$$

if $t=\frac{a}{a+1}$. It is not difficult to check that $1 \leq \Phi\left(x_{1}, \ldots, x_{d}\right) \leq d$, hence $\frac{1}{d+1} \leq$ $1-t \leq \frac{1}{2}$.

Note that $\Phi=\Phi\left(x_{1}, \ldots, x_{d+1}\right)$ can be viewed as a function of $d$ independent indeterminates $x_{1}, \ldots, x_{d}$. Conditions $0<\gamma \leq x_{1}, \ldots, x_{d}$ and $\frac{1}{d+1} \leq x_{d+1} \leq \frac{1}{2}$ define a compact domain $Q$ in $\mathbb{R}^{d}$ since $x_{d+1}=1-x_{1}-\cdots-x_{d}$. Since $\Phi$ is continuous on $Q$ there exists an integer $k$ such that

$$
\mid \Phi\left(x_{1}, \ldots, x_{d}, x_{d+1}-\Phi\left(x_{1}^{\prime}, \ldots, x_{d}^{\prime}, x_{d+1}^{\prime} \mid<\varepsilon\right.\right.
$$

as soon as $\left|x_{i}-x_{i}^{\prime}\right|<\frac{1}{k}$ for all $i=1, \ldots, d$. Clearly, $k$ does not depend on $n$ and $\lambda$. Then there exists a rational number $t_{0}=\frac{q}{k}<1$ such that $\left|t-t_{0}\right|<\frac{1}{k}$ and

$$
\left|\Phi\left(t_{0} z_{1}, \ldots, t_{0} z_{d}, 1-t_{0}\right)-a-1\right|<\varepsilon .
$$

Denote $y_{0}=1-t_{0}$. Then $t_{0} z_{i} \leq 1-t_{0}=y_{0} \leq t_{0} z_{i-1}$ for some $i$ (or $y_{0}>t_{0} z_{1}$, or $\left.y_{0}<t_{0} z_{d}\right)$. 
Now we set $n^{\prime}=k n$,

$$
\begin{aligned}
& \mu_{1}=q \lambda_{1}, \ldots, \mu_{i-1}=q \lambda_{i-1}, \\
& \mu_{i+1}=q \lambda_{i}, \ldots, \mu_{d+1}=q \lambda_{d}
\end{aligned}
$$

and $\mu_{i}=n(k-q)$. Then $\mu=\left(\mu_{1}, \ldots, \mu_{d+1}\right)$ is a partition of $n^{\prime}$ and

$$
\Phi(\mu)=\Phi\left(t_{0} z_{1}, \ldots, t_{0} z_{d}, 1-t_{0}\right) .
$$

In particular, $|\Phi(\lambda)-\Phi(\mu)-1|<\varepsilon$ by (3) and (4), and we have completed the proof of our lemma.

\section{Algebras of infinite Words}

In this section we recall some constructions and algebras from [3] and their properties. These algebras will be used for constructing unital algebras.

Let $K=\left(k_{1}, k_{2}, \ldots\right)$ be an infinite sequence of integers $k_{i} \geq 2$. Then the algebra $A(K)$ is defined by its basis

$$
\{a, b\} \cup Z_{1} \cup Z_{2} \cup \ldots
$$

where

$$
Z_{i}=\left\{z_{j}^{(i)} \mid 1 \leq j \leq k_{i}, i=1,2, \ldots\right\}
$$

with the multiplication table

$$
z_{1}^{(i)} a=z_{2}^{(i)}, \ldots, z_{k_{i}-1}^{(i)} a=z_{k_{i}}^{(i)}, z_{k_{i}}^{(i)} b=z_{1}^{(i+1)}
$$

for all $i=1,2, \ldots$. All remaining products are assumed to be zero.

It is easy to verify (see also [3]) that $A$ satisfies the identity $x_{1}\left(x_{2} x_{3}\right)=0$ and if $m_{\lambda} \neq 0$ in (1) then $\lambda=\left(\lambda_{1}\right)$ or $\lambda=\left(\lambda_{1}, \lambda_{2}\right)$ or $\lambda=\left(\lambda_{1}, \lambda_{2}, 1\right)$. Denote by $W_{n}^{(d)}$, $d \leq n$, the subspace of the free algebra $F\{X\}$ of all homogeneous polynomials of degree $n$ on $x_{1}, \ldots, x_{d}$. Given a PI-algebra $A$, we define

$$
W_{n}^{(d)}(A)=\frac{W_{n}^{(d)}}{W_{n}^{(d)} \cap I d(A)} .
$$

Recall that the height $h(\lambda)$ of a partition $\lambda=\left(\lambda_{1}, \ldots, \lambda_{d}\right)$ is equal to $d$. We will use the following result from [3].

Lemma 4. ([3, Lemma 4.1]) Let $A$ be a PI-algebra with nth cocharacter $\chi_{n}(A)=$ $\sum_{\lambda \vdash n} m_{\lambda} \chi_{\lambda}$. Then for every $\lambda \vdash n$ with $h(\lambda) \leq d$ we have that $m_{\lambda} \leq \operatorname{dim} W_{n}^{(d)}(A)$.

Now let $w=w_{1} w_{2} \ldots$ be an infinite word in the alphabet $\{0,1\}$. Given an integer $m \geq 2$, let $K_{m, w}=\left\{k_{i}\right\}, i=1,2, \ldots$, be the sequence defined by

$$
k_{i}=\left\{\begin{aligned}
m, \text { if } w_{i} & =0 \\
m+1, \text { if } w_{i} & =1
\end{aligned}\right.
$$

and write $A(m, w)=A\left(K_{m, w}\right)$.

Recall that the complexity $C o m p_{w}(n)$ of an infinite word $w$ is the number of distinct subwords of $w$ of the length $n$ (see [12], Chapter 1). Slightly modifying the proof of Lemma 4.2 from [3] we obtain: 
Lemma 5. For any $m \geq 2$ and for any infinite word $w$ the following inequalities hold

and

$$
\operatorname{dim} W_{n}^{(d)}(A(m, w)) \leq d(m+1) n C o m p_{w}(n)
$$

$$
l_{n}(A(m, w)) \leq n^{3} \operatorname{dim} W_{n}^{(3)}(A(m, w)) .
$$

Now we fix the algebra $A(m, w)$ by choosing the word $w$. Obviously, $\operatorname{Comp}_{w}(n) \leq$ $T$ for any infinite periodic word with period $T$. It is known (see [12]) that $C o m p_{w}(n) \geq$ $n+1$ for any aperiodic word $w$. In case $\operatorname{Comp}_{w}(n)=n+1$ for all $n \geq 1$ the word $w$ is said to be Sturmian. It is also known that for any Sturmian or periodic word the limit

$$
\pi(w)=\lim _{n \rightarrow \infty} \frac{w_{1}+\cdots+w_{n}}{n}>0
$$

always exists (we always assume that a periodic word is non-zero). This limit $\pi(w)$ is called the slope of $w$. For any real number $\alpha \in(0,1)$ there exists a word $w$ with $\pi(w)=\alpha$ and $w$ is Sturmian or periodic depending on whether $\alpha$ is irrational or rational, respectively. Moreover,

$$
\exp (A(m, w))=\Phi_{0}(\beta)=\frac{1}{\beta^{\beta}(1-\beta)^{1-\beta}}
$$

for Sturmian or periodic word $w$, where $\beta=\frac{1}{m+\alpha}, \alpha=\pi(w)$ ([3], Theorem 5.1). As a consequence, for any real $1 \leq \alpha \leq 2$ there exists an algebra $A$ such that $\exp (A)=\alpha$.

Finally, for any periodic word $w$ and for any $m \geq 2$ there exists a finite dimensional algebra $B(m, w)$ satisfying the same identities as $A(m, w)$. In particular, for any rational $0<\beta \leq \frac{1}{2}$ there exists a finite dimensional algebra $B$ with

$$
\exp (B)=\Phi_{0}(\beta)=\frac{1}{\beta^{\beta}(1-\beta)^{1-\beta}} .
$$

\section{Algebra With ADJOINED Unit}

We fix an infinite or periodic word $w$ and $m \geq 2$ and consider the algebra $A=A(m, w)$. Denote by $A^{\sharp}$ the algebra obtained from $A$ by adjoining external unit element 1. Our main goal is to prove that $\exp \left(A^{\sharp}\right)$ exists and that

$$
\exp \left(A^{\sharp}\right)=\exp (A)+1 .
$$

First we find a polynomial upper bound for the colength of $A^{\sharp}$. We start with the remark concerning an arbitrary algebra $B$. Recall that, given an algebra $B$, $W_{n}^{(d)}(B)$ is the dimension of the space of homogeneous polynomials on $x_{1}, \ldots, x_{d}$ of total degree $n$ modulo ideal $\operatorname{Id}(B)$.

Lemma 6. Let $B$ be an arbitrary algebra. Suppose that $\operatorname{dim} W_{n}^{(d)}(B) \leq \alpha n^{T}$ for some natural $T, \alpha \in \mathbb{R}$ and for all $n \geq 1$. Then

$$
\operatorname{dim} W_{n}^{(d)}\left(B^{\sharp}\right) \leq \alpha(n+1)^{T+d+1} .
$$

Proof. Denote by $F\{X\}^{\sharp}$ absolutely free algebra generated by $X$ with adjoined unit element. First note that a multihomogeneous polynomial $f\left(x_{1}, \ldots, x_{d}\right)$ is an identity of $B^{\sharp}$ if all multihomogeneous on $x_{1}, \ldots, x_{d}$ components of $f\left(1+x_{1}, \ldots, 1+\right.$ $x_{d}$ ) are identities of $B$. 
Clearly, the number of multihomogeneous polynomials on $x_{1}, \ldots, x_{d}$ of total degree $k$, linearly independent modulo $I d(B)$, does not exceed $\operatorname{dim} W_{k}^{(d)}(B)$. On the other hand, the number of multihomogeneous components of total degree $k$ in a free algebra $F\left\{x_{1}, \ldots, x_{d}\right\}$ does not exceed $(k+1)^{d}$. Take now

$$
N=(k+1)^{d} \sum_{k=0}^{n} \operatorname{dim} W_{k}^{(d)}(B)+1
$$

assuming that $\operatorname{dim} W_{0}^{(d)}(B)=1$. Clearly,

$$
N \leq 1+(n+1)^{d} \alpha \sum_{k=0}^{n} k^{T}<\alpha(n+1)^{T+d+1} .
$$

Given homogeneous polynomials $f_{1}, \ldots, f_{N+1}$ on $x_{1}, \ldots, x_{d}$ of degree $n$, consider their linear combination $f=\lambda_{1} f_{1}+\cdots+\lambda_{N+1} f_{N+1}$ with unknown coefficients $\lambda_{1}, \cdots, \lambda_{N+1}$. The assumption that some multihomogeneous component of $f\left(1+x_{1}, \ldots, 1+x_{d}\right)$ is an identity of $B^{\sharp}$ is equivalent to some linear equation on $\lambda_{1}, \cdots, \lambda_{N+1}$. Hence the condition that all multihomogeneous components of $f\left(1+x_{1}, \ldots, 1+x_{d}\right)$ are identities of $B$ leads to at most $N$ linear equations on $\lambda_{1}, \cdots, \lambda_{N+1}$. It follows that $f, \ldots, f_{N+1}$ are linearly dependent modulo $\operatorname{Id}\left(B^{\sharp}\right)$ and we have completed the proof.

Lemma 7. Let $A=A(m, w)$ where $m \geq 2$ and $w$ is periodic or Sturmian word. Then

$$
l_{n}\left(A^{\sharp}\right) \leq 4(m+1)(n+1)^{12}
$$

for all sufficiently large $n$.

Proof. First note that the cocharacter of $A^{\sharp}$ lies in the strip of width 4 that is, if $m_{\lambda} \neq 0$ in the decomposition

$$
\chi_{n}\left(A^{\sharp}\right)=\sum_{\lambda \vdash n} m_{\lambda} \chi_{\lambda}
$$

then $h(\lambda) \leq 4$. The number of partitions of $n$ of type $\lambda=\left(\lambda_{1}, \ldots, \lambda_{k}\right)$ with $1 \leq k \leq 4$ is less than $n^{4}$. By Lemma 5

$$
\operatorname{dim} W_{n}^{(4)}(A) \leq 4(n+1) \operatorname{Comp}_{w}(n) .
$$

If $w$ is Sturmian word then $\operatorname{Comp}_{w}(n)=n+1$. If $w$ is periodic then its complexity is finite and hence $\operatorname{Comp}_{w}(n) \leq n+1$ for all sufficiently large $n$ in (10). In particular,

$$
\operatorname{dim} W_{n}^{(4)}(A) \leq 4(m+1)(n+1)^{2} \leq 4(m+1) n^{2}
$$

for all sufficiently large $n$. Applying Lemmas 4, 5 and 6 we obtain

$$
m_{\lambda} \leq \operatorname{dim} W_{n}^{(4)}\left(A^{\sharp}\right) \leq 4(m+1)(n+1)^{8}
$$

for all $m_{\lambda} \neq 0$ in (9) and then

$$
l_{n}\left(A^{\sharp}\right)=\sum_{\lambda \vdash n} m_{\lambda} \leq 4(m+1) n^{4}(n+1)^{8} \leq .4(m+1)(n+1)^{12} .
$$

In the next step we shall find an upper bound for $\Phi(\lambda)$ provided that $m_{\lambda} \neq 0$ in the $n$th cocharacter of $A^{\sharp}$. 
Lemma 8. For any $\varepsilon>0$ there exists $n_{0}$ such that $m_{\lambda}=0$ in (9) if $n>n_{0}$ and

$$
\frac{\lambda_{3}}{\lambda_{1}} \geq \frac{\beta}{1-\beta}+\varepsilon
$$

where $\beta=\frac{1}{m+\alpha}$ and $\alpha$ is the slope of $w$.

Proof. First let $\lambda=\left(\lambda_{1}, \lambda_{2}, \lambda_{3}, 1\right) \vdash n$. Inequality $m_{\lambda} \neq 0$ means that there exists a multilinear polynomial $g$ of degree $n$ depending on one alternating set of four variables, $\lambda_{3}-1$ alternating sets of three variables and some extra variables and $g$ is not an identity of $A^{\sharp}$. That is, there exists an evaluation $\varphi: F\{X\}^{\sharp} \rightarrow A^{\sharp}$ such that $\varphi(g) \neq 0$ and the set $\left\{\varphi\left(x_{1}\right), \ldots, \varphi\left(x_{n}\right)\right\}$ contains at least $\lambda_{3}$ basis elements $b \in A$ and at most $\lambda_{1}$ elements $a \in A$. Obviously, $\varphi(g)=0$ if $\left\{\varphi\left(x_{1}\right), \ldots, \varphi\left(x_{n}\right)\right\}$ does not contain exactly one element $z_{j}^{(i)} \in A$.

Any non-zero product of basis elements of $A$ is the left-normed product of the type

$$
z_{j}^{(i)} a^{k_{1}} b^{l_{1}} \cdots a^{k_{t}} b^{l_{t}}
$$

where $k_{1}, \ldots, k_{t}, l_{1}, \ldots, l_{t}$ are equal to 0 or 1 . More precisely, this product can be written in the form

$$
z_{j}^{(i)} f(a, b)
$$

where

$$
f(a, b)=a^{t_{0}} b a^{t_{1}} b \cdots b a^{t_{k}} b a^{t_{k+1}}
$$

is an associative monomial on $a$ and $b$ and

$t_{0}=m+w_{i}-j, t_{1}=m+w_{i+1}-1, \ldots, t_{k}=m+w_{i+k}-1, t_{k+1} \leq m+w_{i+k+1}-1$.

In particular, $\operatorname{deg}_{b} f=k+1$ and

$$
\operatorname{deg}_{a} f=t_{0}+t_{1}+\cdots+t_{k+1} \geq t_{1}+\cdots+t_{k}=(m-1) k+w_{i+1}+\cdots+w_{i+k} .
$$

The total degree of monomial (11) (i.e. the number of factors) is

$$
n=(m+1) k+w_{i}+\cdots+w_{i+k}+t_{k+1}-j+1 .
$$

Hence, $(m+1) k \geq n-(1+k)-m-1$ and $k \geq \frac{n-m-2}{m+2}$. In particular, $k$ grows with increasing $n$.

It is known that

$$
\frac{w_{i+1}+\cdots+w_{i+k}}{k} \geq \alpha-\frac{C}{k}
$$

for some constant $C$ (see [3], Proposition 5.1 or [12], Section 2.2). This implies that

$$
\operatorname{deg}_{a} f>(m-1) k+k(\alpha-\delta)
$$

where $\delta=\frac{C}{k}$ and

$$
\frac{\operatorname{deg}_{b} f}{\operatorname{deg}_{a} f}<\frac{1+\frac{1}{k}}{m-1+\alpha-\delta} .
$$

Since $\varphi(g) \neq 0$, at least one monomial of the type (11) in $\varphi(g)$ is non-zero. Therefore

$$
\frac{\lambda_{3}}{\lambda_{1}} \leq \frac{\operatorname{deg}_{b} f}{\operatorname{deg}_{a} f}<\frac{1+\frac{1}{k}}{m-1+\alpha-\delta} .
$$

Since $\delta$ is an arbitrary small positive real number, one can choose $n_{0}$ such that

$$
<\frac{1+\frac{1}{k}}{m-1+\alpha-\delta}<\frac{1}{m-1+\alpha}+\frac{\varepsilon}{2}
$$


for all $n \geq n_{0}$. Combining (12) and (13) we conclude that

$$
\frac{\lambda_{3}}{\lambda_{1}}<\frac{1}{m-1+\alpha}+\frac{\varepsilon}{2}
$$

provided that $m_{\lambda} \neq 0$ in (9) and $n \geq n_{0}$. Note that $\frac{\beta}{1-\beta}=\frac{1}{m-1+\alpha}$, hence we have completed the proof of our lemma in case $\lambda=\left(\lambda_{1}, \lambda_{2}, \lambda_{3}, 1\right)$.

Slightly modifying previous arguments we get the proof of the inequality (14) for a partition $\lambda=\left(\lambda_{1}, \lambda_{2}, \lambda_{3}\right)$ with three parts. The only difference is that nonidentical polynomial $g$ depends on at least $\lambda_{3}$ skewsymmetric sets of variables of order 3 but after evaluation, one of these variables can be replaced by $z_{j}^{(i)}$ and we get the inequality

$$
\frac{\lambda_{3}-1}{\lambda_{1}} \leq \frac{\operatorname{deg}_{b} f}{\operatorname{deg}_{a} f}
$$

instead of (12). Taking into account that $\lambda_{1} \rightarrow \infty$ if $n \rightarrow \infty$ we get the same conclusion and thus complete the proof.

For the lower bound of codimensions of $A^{\sharp}$ we need the following results.

Let $A=A(m, w)$ be an algebra defined by an integer $m \geq 2$ and by an infinite word $w=w_{1} w_{2} \ldots$ in the alphabet $\{0,1\}$. Then

$$
z_{1}^{(1)} a^{i_{1}} b a^{i_{2}} b \cdots a^{i_{r}} b=z_{1}^{r+1}
$$

if $i_{1}=m-1+w_{1}, i_{2}=m-1+w_{2}, \ldots, i_{r}=m-1+w_{r}$. Otherwise the left hand side of (15) is zero.

Lemma 9. Let $\lambda=\left(j, \lambda_{2}, \lambda_{3}, 1\right)$ be a partition of $n=j+m r+w_{1}+\cdots+w_{r}+1$ with $j \geq \lambda_{2}=(m-1) r+w_{1}+\cdots+w_{r}, \lambda_{3}=r$ or let $\lambda=\left(\lambda_{1}, j, \lambda_{3}, 1\right)$ be a partition of the same $n$ with $\lambda_{1}=(m-1) r+w_{1}+\cdots+w_{r}>j \geq \lambda_{3}=r$. Then $m_{\lambda} \neq 0$ in (9).

Proof. Recall that, given $S_{n}$-module $M$, the multiplicity of $\chi_{\lambda}$ in the character $\chi(M)$ is non-zero if $e_{T_{\lambda}} M \neq 0$ for some Young tableaux $T_{\lambda}$ of shape $D_{\lambda}$. The essential idempotent $e_{T_{\lambda}} \in F S_{n}$ is equal to

$$
e_{T_{\lambda}}=\left(\sum_{\sigma \in R_{T_{\lambda}}} \sigma\right)\left(\sum_{\tau \in C_{T_{\lambda}}}(\operatorname{sgn} \tau) \tau\right. \text {. }
$$

Here $R_{T_{\lambda}}$ is the row stabilizer of $T_{\lambda}$, i.e. the subgroup of all $\sigma \in S_{n}$ permuting indices only inside rows of $T_{\lambda}$ and $C_{T_{\lambda}}$ is the column stabilizer of $T_{\lambda}$.

First let $\lambda_{1}=j \geq \lambda_{2}$. Denote $n_{0}=m r+w_{1}+\cdots+w_{r}+1$ and consider the Young tableaux $T_{\lambda}$ of the following type. Into the boxes of the 1st row of $D_{\lambda}$ we place $n_{0}+1, \ldots, n_{0}+j$ from left to right. Into the third row we insert $j_{1}=i_{1}+2, \ldots, j_{r}=$ $i_{1}+\cdots+i_{r}+r+1$. (In fact, $j_{1}, \ldots, j_{r}$ are the positions of $b$ in the product (15)). Into the second row we insert from left to right $j_{1}-1, \ldots, j_{r}-1, i_{r+1}, \ldots, i_{\lambda_{2}}$ where $\left\{i_{r+1}, \ldots, i_{\lambda_{2}}\right\}=\left\{2, \ldots, n_{0}\right\} \backslash\left\{j_{1}-1, j_{1}, \ldots, j_{r}-1, j_{r}\right\}$ and into the unique box of the 4 th row we put 1 .

Then

$$
e_{T_{\lambda}}\left(x_{1}, \ldots, x_{n}\right)=\operatorname{Sym}_{1} \operatorname{Sym}_{2} \operatorname{Sym}_{3} A l t_{1} \cdots A l t_{\lambda_{2}}\left(x_{1}, \ldots, x_{n}\right)
$$

where

- $A l t_{1}$ is the alternation on $\left\{1, j_{1}-1, j_{1}, n_{0}+1\right\}$;

- $A l t_{k}$ is the alternation on $\left\{j_{k}-1, j_{k}, n_{0}+k\right\}$ if $2 \leq k \leq r$;

- Alt $_{k}$ is the alternation on $\left\{i_{k}, n_{0}+k\right\}$ if $r<k \leq \lambda_{2}$; 
- $\operatorname{Sym}_{1}$ is the symmetrization on $\left\{n_{0}+1, \ldots, n_{0}+j\right\}$;

- $\operatorname{Sym}_{2}$ is the symmetrization on $\left\{j_{1}, \ldots, j_{r}\right\}$;

- $\operatorname{Sym}_{3}$ is the symmetrization on $\{2, \ldots, n\} \backslash\left\{j_{1}, \ldots, j_{r}\right\}$.

After an evaluation

$$
\varphi\left(x_{1}\right)=z_{1}^{(1)}, \varphi\left(x_{n_{0}+1}\right)=\cdots=\varphi\left(x_{n_{0}+j}\right)=1 \in A^{\sharp}, \varphi\left(x_{j_{1}}\right)=\cdots=\varphi\left(x_{j_{r}}\right)=b
$$

and

$$
\varphi\left(x_{i}\right)=a \text { if } i \neq 1, j_{1}, \ldots, j_{r}, n_{0}+1, \ldots, n_{0}+j
$$

we have

$$
\varphi\left(e_{T_{\lambda}}\left(x_{1} \cdots x_{n}\right)\right)=j ! r !\left(n_{0}-r-1\right) ! z_{1}^{(r+1)} \neq 0,
$$

hence $m_{\lambda} \neq 0$ in (9).

Similarly, filling up the second row of $T_{\lambda}$ by $n_{0}+1, \ldots, n_{0}+j$ in case $\lambda_{1}=$ $(m-1)+w_{1}+\cdots+w_{r}>j \geq \lambda_{3}=r$ we prove that $e_{T_{\lambda}}\left(x_{1} \cdots x_{n}\right)$ is not an identity of $A^{\sharp}$.

Recall that, given $0 \leq \beta \leq 1$,

$$
\Phi_{0}(\beta)=\Phi(\beta, 1-\beta)=\frac{1}{\beta^{\beta}(1-\beta)^{1-\beta}} .
$$

Lemma 10. Let $A=A(m, w)$ be an algebra defined for an integer $m \geq 2$ and $a$ Sturmian or periodic word $w$ with slope $\alpha$. Let also $\beta=\frac{1}{m+\alpha}$. Then for any $\varepsilon>0$ there exist a constant $C$, positive integers $n_{1}<n_{2} \ldots$ and partitions $\lambda^{(i)} \vdash n_{i}$ such that for some large enough $i_{0}$ the following properties hold:

1) $\left|\Phi\left(\lambda^{(i)}\right)-\Phi_{0}(\beta)-1\right|<\varepsilon$ for all $i \geq i_{0}$;

2) $n_{i+1}-n_{i}<C$ for all $i \geq i_{0}$;

3) $m_{\lambda}^{(i)} \neq 0$ in $\chi_{n_{i}}\left(A^{\sharp}\right)$ for all $i \geq i_{0}$.

Proof. Note that $\beta<\frac{1}{2}$ since $\alpha>0$. First take an arbitrary $r \geq 1, n=$ $m r+w_{1}+\cdots+w_{r}$ and $\lambda=\left(\lambda_{1}, \lambda_{2}\right)$, where $\lambda_{1}=(m-1) r+w_{1}+\cdots+w_{r}, \lambda_{2}=r$. We set

$$
\begin{gathered}
x_{1}=\frac{\lambda_{1}}{n}=\frac{m-1+\frac{w_{1}+\cdots+w_{r}}{r}}{m+\frac{w_{1}+\cdots+w_{r}}{r}}, \\
x_{2}=\frac{\lambda_{2}}{n}=\frac{1}{m+\frac{w_{1}+\cdots+w_{r}}{r}} .
\end{gathered}
$$

As it was mentioned in the proof of Lemma 8 (see also [3], Proposition 5.1 or [12], Section 2.2) there exists a constant $C_{1}$ such that

$$
\left|\frac{w_{1}+\cdots+w_{r}}{r}-\alpha\right|<\frac{C_{1}}{r} .
$$

Hence for any $\varepsilon_{1}>0$ we can find $r_{0}$ such that

$$
\left|\Phi(\lambda)-\Phi_{0}(\beta)\right|<\varepsilon_{1}
$$

for all $r \geq r_{0}$ since $\Phi\left(z_{1}, z_{2}\right)$ is a continuous function and $\left(x_{1}, x_{2}\right) \rightarrow(1-\beta, \beta)$ when $r \rightarrow \infty$.

Now using Lemma 2 and Lemma 3, given $\varepsilon_{2}>0$, we insert one extra row into $D_{\lambda}$ that is, we construct a partition $\mu=\left(\mu_{1}, \mu_{2}, \mu_{3}\right)$ of $n_{0}=n k$ such that

$$
|\Phi(\lambda)-\Phi(\mu)-1|<\varepsilon_{2} .
$$


We have three options. Either $\mu_{1}$ is a new row (that is, $\left(\mu_{2}, \mu_{3}\right)=\left(q \lambda_{1}, q \lambda_{2}\right)$ or $\mu_{2}$ is a new row (that is, $\left(\mu_{1}, \mu_{3}\right)=\left(q \lambda_{1}, q \lambda_{2}\right)$ or $\mu_{3}$ is a new row (that is, $\left(\mu_{1}, \mu_{2}\right)=\left(q \lambda_{1}, q \lambda_{2}\right)$.

First we exclude the third case. Suppose that $\left(\mu_{1}, \mu_{2}\right)=\left(q \lambda_{1}, q \lambda_{2}\right)$. Recall that by Lemma 2 the maximal value of $\Phi\left(t z_{1}, t z_{2}, 1-t\right)$ is achieved if

$$
t=\frac{\Phi\left(z_{1}, z_{2}\right)}{1+\Phi\left(z_{1}, z_{2}\right)}
$$

Since $\Phi\left(z_{1}, z_{2}\right)<2$ if $\beta<\frac{1}{2}$ we obtain that $1-t>\frac{1}{3}$. For Lemma 3 this means that the new row of $D_{\mu}$ cannot be the third row that is, case $\left(\mu_{1}, \mu_{2}\right)=\left(q \lambda_{1}, q \lambda_{2}\right)$ is impossible.

Now let $\left(\mu_{2}, \mu_{3}\right)=\left(q \lambda_{1}, q \lambda_{2}\right)$. We exchange $\mu$ to $\mu^{\prime}$ in the following way. We set $\mu_{2}^{\prime}=q r(m-1)+w_{1}+\cdots+w_{q r}$ and take $\mu^{\prime}=\left(\mu_{1}, \mu_{2}^{\prime}, \mu_{3}\right)$. Then $\mu^{\prime} \vdash n^{\prime}$ where

$$
n^{\prime}-n_{0}=\mu_{2}^{\prime}-\mu_{2}=w_{1}+\cdots+w_{q r}-q\left(w_{1}+\cdots+w_{r}\right) .
$$

Using again inequality (16) we get

$$
\left|n^{\prime}-n_{0}\right|<C_{1}(q+1) \text {. }
$$

Inequality (19) also shows that $\mu_{1} \geq \mu_{2}^{\prime} \geq \mu_{3}$ if $n$ is sufficiently large and our construction of partition $\mu$ is correct.

Clearly, $\left|\Phi(\mu)-\Phi\left(\mu^{\prime}\right)\right| \rightarrow 0$ if $n \rightarrow \infty$ and

$$
\left|\Phi(\mu)-\Phi\left(\mu^{\prime}\right)\right|<\varepsilon_{3}
$$

for any fixed $\varepsilon_{3}>0$ for all sufficiently large $r$ (and $n$ ). Starting from this sufficiently large $r$ we denote $n_{r}=n^{\prime}+1$ and take $\lambda^{(r)} \vdash n_{r}, \lambda^{(r)}=\left(\mu_{1}, \mu^{\prime}, \mu_{3}, 1\right)$. All preceding $n_{1}, \ldots, n_{r-1}$ and $\lambda^{(1)}, \ldots, \lambda^{(r-1)}$ we choose in an arbitrary way.

Since $\mu_{3}=q r$, by Lemma 9 the multiplicity of the irreducible character $\lambda^{(r)}$ in $\chi_{n_{r}}\left(A^{\sharp}\right)$ is not equal to zero and $\left|n_{r}-k n\right|<C_{2}=C_{1}(q+1)+1$ by (19) since $n_{0}=n k$. It is not difficult to see that in this case

$$
\left|\Phi\left(\mu^{\prime}\right)-\Phi\left(\lambda^{(r)}\right)\right|<\varepsilon_{4}
$$

for any fixed $\varepsilon_{4}>0$ if $r$ (and the corresponding $n$ ) is sufficiently large. Combining (17), (18), (20) and (21) we see that $\lambda^{(r)}$ satisfies conditions 1) and 3) of our lemma. Finally, consider the difference between $n_{r}$ and $n_{r+1}$ provided that all $n_{r+1}, n_{r+2}, \ldots$ are constructed by the same procedure. That is, we take

$$
\bar{n}=m(r+1)+w_{1}+\cdots+w_{r+1}+1
$$

and obtain $n_{r+1}$ satisfying the same condition

$$
\left|n_{r+1}-k \bar{n}\right|<C_{2} .
$$

On the other hand, $\bar{n}-k n=k\left(m+w_{r+1}\right) \leq k(m+1)$ and $\left|k n-n_{r}\right|<C_{2}$. Hence we have

$$
\left|n_{r+1}-n_{r}\right|<C=2 C_{2}+k(m+1) .
$$

This latter inequality completes the proof of our lemma if $\left(\mu_{2}, \mu_{3}\right)=\left(q \lambda_{1}, q \lambda_{2}\right)$. Arguments in the case $\left(\mu_{1}, \mu_{3}\right)=\left(q \lambda_{1}, q \lambda_{2}\right)$ are the same. 


\section{The MAIN RESUlT}

Now we are ready to prove the main result of the paper.

Theorem 1. Let $w=w_{1} w_{2} \ldots$ be Sturmian or periodic word and let $A=A(m, w)$, $m \geq 2$, be an algebra defined by $m$ and $w$ in (5) - (8). If $A^{\sharp}$ is the algebra obtained from $A$ by adojining an external unit then PI-exponent of $A^{\sharp}$ exists and

$$
\exp \left(A^{\sharp}\right)=1+\exp (A) .
$$

Proof. Let $\alpha=\pi(w)$ be the slope of $w$ and let $\beta=\frac{1}{m+\alpha}$. Recall that $\exp (A)=$ $\Phi_{0}(\beta)$ where

$$
\Phi_{0}(\beta)=\frac{1}{\beta^{\beta}(1-\beta)^{1-\beta}}
$$

([3]). First we prove that for any $\delta>0$ there exists $N$ such that

$$
\Phi(\lambda)<\Phi_{0}(\beta)+1+\delta
$$

as soon as $\lambda$ is a partition of $n \geq N$ with $m_{\lambda} \neq 0$ in $\chi_{n}\left(A^{\sharp}\right)$.

By Lemma 8 , for any $\varepsilon>0$ there exists $n_{0}$ such that

$$
\frac{\lambda_{3}}{\lambda_{1}}<\frac{\beta}{1-\beta}+\varepsilon
$$

if $n \geq n_{0}, \lambda \vdash n$ and $m_{\lambda} \neq 0$. If $\lambda=(n)$ or $\lambda=\left(\lambda_{1}, \lambda_{2}\right)$ then by the hook formula for dimensions of irreducible $S_{n}$-representations it follows that $\operatorname{deg} \chi_{\lambda} \leq 2^{n}$. Then by Lemma 1

$$
\Phi(\lambda) \leq 2 \sqrt[n]{n^{6}}
$$

and (22) holds for all sufficiently large $n$ since $1 \leq \Phi_{0}(\beta) \leq 2$.

Let $\lambda=\left(\lambda_{1}, \lambda_{2}, \lambda_{3}\right)$. Denote $\mu=\left(\lambda_{1}, \lambda_{3}\right) \vdash n^{\prime}$, where $n^{\prime}=n-\lambda_{2}$. If $x_{1}=$ $\frac{\lambda_{1}}{n^{\prime}}, x_{2}=\frac{\lambda_{3}}{n^{\prime}}$ then

and

$$
\Phi(\mu)=\Phi\left(x_{1}, x_{2}\right)=\Phi_{0}\left(x_{2}\right)
$$

$$
x_{2} \leq \varphi(\varepsilon)=\frac{\beta+(1-\beta) \varepsilon}{1+(1-\beta) \varepsilon}
$$

as follows from (23). Since

$$
\lim _{n \rightarrow \infty} \varphi(\varepsilon)=\beta
$$

and $\Phi_{0}$ is continuous, there exist $N$ and $\varepsilon$ such that $\Phi(\mu)<\Phi_{0}(\beta)+\delta$ for all $n>N$. Then by Lemma 2

$$
\Phi(\lambda) \leq \Phi(\mu)+1<\Phi_{0}(\beta)+1+\delta .
$$

Now consider the case $\lambda=\left(\lambda_{1}, \lambda_{2}, \lambda_{3}, 1\right)$. Excluding the second row of diagram $D_{\lambda}$ we get a partition $\mu=\left(\mu_{1}, \mu_{2}, 1\right)=\left(\lambda_{1}, \lambda_{3}, 1\right)$ of $n^{\prime}=n-\lambda_{2}$ with

$$
\frac{\mu_{2}}{\mu_{1}}<\frac{\beta}{1-\beta}+\varepsilon
$$

Consider also partition $\mu^{\prime}=\left(\mu_{1}, \mu_{2}\right)$ of $n^{\prime}-1$. As before, given $\delta>0$, one can find $n_{0}$ such that

provided that $n \geq n_{0}$.

$$
\Phi\left(\mu^{\prime}\right)<\Phi_{0}(\beta)+\frac{\delta}{2}
$$

Since $\Phi$ is continuous, for all sufficiently large $n$ (and $n^{\prime}$ ) we have

$$
\Phi(\mu)<\Phi_{0}(\beta)+\delta .
$$


Applying again Lemma 2 we get (22). It now follows from (22), Lemmas 1 and 7 that

$c_{n}\left(A^{\sharp}\right)=\sum_{\lambda \vdash n} m_{\lambda} \operatorname{deg} \chi_{\lambda} \leq\left(\Phi_{0}(\beta)+1+\delta\right)^{n} l_{n}\left(A^{\sharp}\right) \leq 4(m+1)(n+1)^{13}\left(\Phi_{0}(\beta)+1+\delta\right)^{n}$.

Hence

$$
\overline{\exp }\left(A^{\sharp}\right)=\limsup _{n \rightarrow \infty} \sqrt[n]{c_{n}\left(A^{\sharp}\right)} \leq \Phi_{0}(\beta)+\delta+1
$$

for any $\delta>0$ that is,

$$
\overline{\exp }\left(A^{\sharp}\right) \leq \Phi_{0}(\beta)+1=\exp (A)+1 .
$$

Now we find a lower bound for codimensions. Since

$$
c_{n}\left(A^{\sharp}\right) \geq \operatorname{deg} \chi_{\lambda} \geq \frac{\Phi(\lambda)^{n}}{n^{20}}
$$

by Lemma 1 if $m_{\lambda} \neq 0$ in $\chi_{n}\left(A^{\sharp}\right)$, then by Lemma 10 for any $\varepsilon>0$ there exists a sequence $n_{1}<n_{2}<\ldots$ such that

$$
c_{n_{i}}\left(A^{\sharp}\right) \geq \frac{1}{n_{i}^{20}}\left(\Phi_{0}(\beta)+1-\varepsilon\right)^{n_{i}}, i=1,2, \ldots
$$

and $n_{i+1}-n_{i}<C=$ const for all $i \geq 1$. Note that the sequence $\left\{c_{n}(R)\right\}$ is non-decreasing for any unital algebra $R$. Then

$$
\underline{\exp }\left(A^{\sharp}\right)=\liminf _{n \rightarrow \infty} \sqrt[n]{c_{n}\left(A^{\sharp}\right)} \geq \Phi_{0}(\beta)+1 .
$$

Now (24) and (25) complete the proof of the theorem.

Corollary 1. For any real numbers $\gamma \in[2,3]$ there exists an algebra $A$ with 1 such that $\exp (A)=\gamma$.

As it was mentioned in the preliminaries, PI-exponents of finite dimensional algebras form a dense subset in the interval $[1,2]$. Hence we get the following

Corollary 2. For any real numbers $\beta<\gamma \in[2,3]$ there exists a finite dimensional algebra $B$ with 1 such that $\beta \leq \exp (B) \leq \gamma$. In particular, PI-exponents of finite dimensional unital algebras form a dense subset in the interval $[2,3]$.

Acknowledgements. This research was supported by the Slovenian Researcg Agency grants P1-0292, J1-4144, and J1-5435, and the Russian Federation for Basic Research grant No 13-01-00234a.

\section{REFERENCES}

[1] O.E. Bezushchak, A.A. Belyaev, M.V. Zaicev, Exponents of identities of algebras with adjoined unit, Vestnik Kiev Nat. Univ. Ser. Phis.-Mat. Nauk, (2012), no 3, 7-9.

[2] O.E. Bezushchak, A.A. Belyaev, M.V. Zaicev, Codimensions of identities of algebras with adjoined unit, Fundam. Prikl. Mat., to appear.

[3] A. Giambruno, S. Mishchenko, M. Zaicev, Codimensions of algebras and growth functions, Adv. Math. 217 (2008) 1027-1052.

[4] A. Giambruno, S. Mishchenko, M. Zaicev, Polynomial identities of algebras of small dimension, Comm. Algebra 37 (2009) 1934-1948.

[5] A. Giambruno, I. Shestakov, M. Zaicev, Finite-dimensional non-associative algebras and codimension growth, Adv. Appl. Math. 47 (2011) 125-139. 
[6] A. Giambruno, M. Zaicev, On codimension growth of finitely generated associative algebras, Adv. Math. 140 (1998) 145-155.

[7] A. Giambruno, M. Zaicev, Exponential codimension growth of PI algebras: an exact estimate, Adv. Math. 142 (1999) 221-243.

[8] A. Giambruno, M. Zaicev, Polynomial Identities and Asymptotic Methods, Math. Surveys Monogr., vol. 12, Amer. Math. Soc., Providence, RI, 2005.

[9] A. Giambruno, M. Zaicev, Proper identities, Lie identities and exponential codimension growth, J. Algebra 320 (2008) 1933-1962.

[10] A. Giambruno, M. Zaicev, On codimension growth of finite-dimensional Lie superalgebras, J. Lond. Math. Soc. (2) 85 (2012) 534-548.

[11] A.R. Kemer, T-ideals with exponential codimension growth are Specht, Sib. Mat. Zh. 19 (1978) 54-69.

[12] M. Lothaire, Algebraic Combinatorics on Words, Encyclopedia Math. Appl., vol. 90. Cambridge University Press, Cambridge, 2002.

[13] S.P. Mishchenko, Lower bounds on the dimensions of irreducible representations of symmetric groups and the exponents of varieties of Lie algebras, Mat. Sb. 187 (1996) 83-94.

[14] M.V. Zaitsev, Integrality of exponents of growth of identities of finite-dimensional Lie algebras. (Russian) Izv. Ross. Akad. Nauk Ser. Mat. 66 (2002) 23-48; English transl.: Izv. Math. 66 (2002) 463-487.

[15] M.V. Zaitsev, Identities of finite-dimensional unital algebras, (Russian) Algebra Logika 50 (2011) 563-594, 693, 695; English transl.: Algebra Logic 50 (2011) 381-404.

Dušan Repovš, Faculty of Education, and Faculty of Mathematics and Physics, University of Lujubljana \& Institute of Mathematics, Physics and Mechanics, Ljubljana, 1000, SLOVEnia

E-mail address: dusan.repovs@guest.arnes.si

Mikhail Zaicev, Department of Algebra, Faculty of Mathematics and Mechanics, Moscow State University, Moscow, 119992, Russia

E-mail address: zaicevmv@mail.ru 